\title{
The Power of Suggestion
}

Broadly speaking, clinical decision support has always existed in medicine through tools clinicians create that help them make accurate, reliable, and efficient decisions. In the modern era, a clinical decision support system (CDSS) refers to "any electronic system designed to aid directly in clinical decision making, in which characteristics of individual patients are used to generate patientspecific assessments or recommendations that are then presented to clinicians for consideration." 1

In this issue of ResPiratory CARE, Bagga et $\mathrm{al}^{2}$ report on their use of a computerized prompt given to physicians entering ventilator orders for patients receiving volume controlled mechanical ventilation, and the impact that this intervention may have had on initial tidal volumes $\left(\mathrm{V}_{\mathrm{T}}\right)$ set by respiratory therapists at a tertiary care academic medical center. The intervention was a CDSS prompt (an on screen pop-up window) that appeared anytime a physician entered orders for mechanical ventilation. The prompt displayed the patient's height, actual weight, and ideal body weight; 3 statements about the average, initial, and ideal $\mathrm{V}_{\mathrm{T}}$ for patients with/without acute lung injury; and 2 calculated $\mathrm{V}_{\mathrm{T}}$ values for 6 and $8 \mathrm{~mL} / \mathrm{kg}$ of ideal body weight. Physicians entering orders were required to acknowledge the information provided by clicking on an OK button, which closed the window. The ordering provider could then order any $\mathrm{V}_{\mathrm{T}}$ of his choosing. The authors retrospectively compared 240 subjects before and after implementation of their tool. Twenty subjects were selected from respiratory care records that equally represented 6 adult ICUs in the before/after periods. ICUs were medically or surgically focused with general, cardiac, or neurologic patient populations.

In their review, Bagga et $\mathrm{al}^{2}$ found a significant decrease in the initial delivered $\mathrm{V}_{\mathrm{T}}$ for subjects in two thirds of the individual ICUs and in their combined cohort (average decrease of $0.84 \mathrm{~mL} / \mathrm{kg}$ of ideal body weight,

\footnotetext{
The opinions or assertions contained herein are the private views of the authors and are not to be construed as official or as reflecting the views of the Department of the Army, the Department of Defense, or the United States Government.
}

The authors have disclosed no conflicts of interest.

DOI: $10.4187 /$ respcare. 03498
$P<.001)$. They also found a significant decrease in the number of subjects ventilated at $>10 \mathrm{~mL} / \mathrm{kg}$ of ideal body weight in all of the ICUs following the introduction of this tool $(20 \%$ vs $4 \%, P=.003)$. Finally, they observed a reduction in variance of the delivered $\mathrm{V}_{\mathrm{T}}$ in two thirds of the ICUs.

Unfortunately, it is impossible to tell from the retrospective study design and data provided if the intervention played any role in the observed reduction in $\mathrm{V}_{\mathrm{T}}$. The authors did not provide evidence that the $\mathrm{V}_{\mathrm{T}}$ ordered by the physician was the same as the $\mathrm{V}_{\mathrm{T}}$ set by the respiratory therapist or that these changes occurred at the same time as implementation of the new CDSS. Without these data, a direct effect of the CDSS on ventilator $\mathrm{V}_{\mathrm{T}}$ has not been proven. The data presented reflect only an association between CDSS implementation and a change in clinical practice.

\section{See the Original Study on Page 1172}

If the authors' institution is similar to most ICUs, respiratory therapists play a significant role in setting the initial $\mathrm{V}_{\mathrm{T}}$, and these settings may or may not reflect a physician's order. There is often not enough time for the physician to write the order or for the respiratory therapist to review it. Thus, the initial $\mathrm{V}_{\mathrm{T}}$ recorded in respiratory therapy documentation likely reflects the knowledge and preferences of the respiratory therapist and not the physician's orders. In this case, other interventions (eg, respiratory therapists' education and training) may have impacted the results of this study, independently of the CDSS implementation.

However, these limitations do not detract from the probable impact of the CDSS that Bagga et $\mathrm{al}^{2}$ implemented. It is probable that implementation of the CDSS raised physician and, subsequently, respiratory therapist awareness of setting the appropriate $\mathrm{V}_{\mathrm{T}}$. Thus, the CDSS may not have had a direct impact on the initial $\mathrm{V}_{\mathrm{T}}$ setting but may have changed the institutional culture of how to manage ventilatory settings by raising awareness of the problem of ordering too high a $\mathrm{V}_{\mathrm{T}}$. Ultimately, the tool may have helped change human behavior in a positive fashion. This is similar to the effect of checklists on human behavior, ${ }^{3,4}$ and it is why clinicians continue to create and use tools to support their decision making. 
Jeremy C Pamplin MD

Leopoldo C Cancio MD

United States Army Institute of Surgical Research

Joint Base San Antonio

Fort Sam Houston, Texas

\section{REFERENCES}

1. Lobach D, Sanders GD, Bright TJ, Wong A, Tech A, Dhurjati R, et al. Enabling health care decisionmaking through clinical decision support and knowledge management. Evidence Report No. 203. Agency for Healthcare Research and Quality Publication No. 12-
E001-EF. Rockville, Maryland: Agency for Healthcare Research and Quality; 2012:1-784.

2. Bagga S, Paluzzi DE, Chen CY, Riggio JM, Nagaraja M, Marik PE, Baram M. Improved compliance with lower tidal volumes for initial ventilatory setting using a computerized clinical decision support system. Respir Care 2014;59(8):1172-1177.

3. Weiss CH, Moazed F, McEvoy CA, Singer BD, Szleifer I, Amaral LA, et al. Prompting physicians to address a daily checklist and process of care and clinical outcomes: a single-site study. Am J Respir Crit Care Med 2011;184(6):680-686.

4. Newkirk M, Pamplin JC, Kuwamoto R, Allen DA, Chung KK. Checklists change communication about key elements of patient care. J Trauma Acute Care Surgery 2012;73(2 Suppl 1):S75-S82. 